\title{
Routine practice needs shifting from linear to purse-string skin closure in closure of stoma sites
}

\author{
Shahab Hajibandeh ${ }^{1}$ (D) $\cdot$ Shahin Hajibandeh ${ }^{2} \cdot$ Andrew Maw $^{1}$ \\ Accepted: 16 January 2020 / Published online: 22 January 2020 \\ (C) Springer-Verlag GmbH Germany, part of Springer Nature 2020
}

\section{Dear Editor:}

We read with interest the article by Neumann et al. [1] published online in December 2019 in International Journal of Colorectal Disease. The authors present results of a retrospective cohort study and systematic review with meta-analysis of the best available evidence (including the results of their cohort study) highlighting that primary skin closure with use of a subcutaneous suction drain reduces the risk of wound healing complications following stoma reversal.

We know from the available evidence that primary skin closure at the stoma reversal site with or without a subcutaneous drain is associated with higher risk of surgical site infection (SSI) in comparison with purse-string skin closure [2]. Although primary skin closure with use of a subcutaneous drain resulted in lower risk of SSI $(8.6 \%)$ compared with primary skin closure without a drain $(15.0 \%)$ in the study by Neumann et al. ${ }^{1}$, the reported risks are higher than the risk of SSI associated with purse-string skin closure (2.5\%) [2]. Whether the use of the purse-string skin closure technique together with a subcutaneous drain results in a still lower risk of SSI in comparison with purse-string skin closure without a drain remains unanswered.

We are in agreement with Neumann et al. [1] that on occasions, purse-string skin closure may not be possible when the length of incision needs to be increased in removal of stoma and where a purse-string closure is not possible for various reasons. In such circumstances, primary skin closure with a subcutaneous drain may be the closure technique of choice.

Shahab Hajibandeh

shahab_hajibandeh@yahoo.com

1 Department of Colorectal and General Surgery, Glan Clwyd Hospital, Rhyl, UK

2 Department of General Surgery, Sandwell and West Birmingham Hospitals NHS Trust, West Bromwich, UK
Unlike Neumann et al. [1], we do not think that purse-string skin closure technique is increasingly utilized during stoma reversal. We conducted a multicenter observational study enrolling 593 patients undergoing reversal of stoma in 4 centers in the UK (three centers in the North West of England and one center in North Wales), and our results suggested that in only $11 \%$ of cases, the stoma closure site was closed by purse-string closure. This highlights that despite the fact that the best available evidence suggests that purse-string closure should be the skin closure technique of choice in patients undergoing stoma reversal, many surgeons still use the linear skin closure technique. There is a need for a consensus to consider purse string closure as the skin closure technique of choice in order to shift the clinical practice from linear closure to purse-string closure.

We would like to congratulate the authors for their work and look forward to future studies by Neumann et al. and other authors to further clarify this area of practice.

\section{Compliance with ethical standards}

Conflict of interest The authors declare that they have no conflict of interest.

Ethical approval Not required.

\section{References}

1. Neumann PA, Reischl S, Berg F, Jäger C, Friess H, Reim D et al (2019 Dec) Meta-analysis and single-center experience on the protective effect of negative suction drains on wound healing after stoma reversal. Int J Color Dis 24

2. Hajibandeh S, Hajibandeh S, Kennedy-Dalby A, Rehman S, Zadeh RA (2018 Oct) Purse-string skin closure versus linear skin closure techniques in stoma closure: a comprehensive meta-analysis with trial sequential analysis of randomised trials. Int J Color Dis 33(10):1319-1332

Publisher's note Springer Nature remains neutral with regard to jurisdictional claims in published maps and institutional affiliations. 